\title{
Gestión de información sat río Manzanares
}

\author{
Information management SAT Rio Manzanares
}

\author{
Viloria, Adolfo; Quintero, Wilmer \\ adolfo.viloriac@campusucc.edu.co, \\ Wilmer.quintero@campusucc.edu.co. \\ Universidad Cooperativa de Colombia UCC
}




\section{Resumen}

$\mathrm{E}$ 1 siste de datos consiste de un modulo de lectura de las variables humedad del suelo, nivel de lámina de agua y precipitación. El prototipo tiene como base un Arduino y una interfaz gráfica que muestra las lecturas en tiempo real de los datos obtenidos de los sensores. Para la transmisión de la información se ha implementado una red Mesh con la tecnología Zigbee como sistema de respaldo para el envío de datos. Por ultimo contamos con un portal web que gestiona la red de sensores, analiza la información medida y emite la alerta a los usuarios por medio de una App Android.
SAT temprana, sensores, App, Sistema de información.

\footnotetext{
Abstract

The system of information developed for collecting and processing data consists of a reading module for the humidity variables of the soil, level of water and precipitation. The prototype has as a basis an Arduino and a graphic interfase that shows the readings in real time of the data obtained by the sensors.For the transmission of information a Mesh Keywords: words: SAT temprana, sensors, App, Information system. net has been implemented with a Zigbee technology as a backing system for sending the data. Finally, we rely on a portal web that manages a net of sensors, analyses the measured information and issues a warning to users through an App Android.
} 
Introducción

L os Sistemas de Alerta Temprana (SAT) juegan un papel importante en la gestión del riesgo de desastres en los países de América, como se entiende en el manual presentado por la OEA [1]. No obstante, también existen mecanismos de entes no gubernamentales como el Banco Mundial, que maneja el Global Facility For Disaster Reduction and Recovery (GFDRR). En conjunto con el departamento de desarrollo sostenible para los países europeos y Asia Central, el Banco Mundial [2] posee un estudio en el que se consideran dos aspectos claves al respecto: 1. Los pronósticos de las condiciones de peligro por riesgo ante desastres relacionados con el clima, 2. la provisión de las medidas para mitigar y manejar tales riesgos.

En diversas universidades se han trabajado proyectos de investigación con el enfoque de sistemas de alerta temprana. Entre estos trabajos se encuentra el de Krzhizhanovskaya [3], que presentan un sistema de soporte de decisiones para la gestión de desastres asociados a inundaciones. Este sistema incluye modelos para predicciones meteorológicas y simulación de la presión atmosférica, viento, olas del mar y sus ondas estacionarias. También incluye un módulo de optimización de la operación de compuertas hidráulicas, así como modelos para el estudio de la estabilidad de diques y muros de contención. Los autores simulan las dinámicas de inundación y diferentes escenarios de evacuación.

En otros trabajos desarrollados sobre el tema, los autores proponen un modelo de referencia y metodología para construir sistemas de alerta temprana, teniendo en cuenta los cuatro ciclos propios de un
Sistema de Alerta Temprana, que son: (1) Monitoreo: donde se almacenan los datos de los sensores en tiempo real, (2) Análisis: donde una o más aplicaciones realizan un procesamiento y análisis de los datos, tales como detección anormal y simulaciones basadas en sistemas expertos, (3) Juicio: donde los resultados de los análisis son identificados según las reglas específicas del Sistema de Alerta Temprana (SAT) para determinar el nivel de riesgo, (4) Aviso/Acto: ciclo en el que el SAT informa sobre recomendaciones específicas o, si es necesario, la realización de acciones según los niveles de riesgo determinados $[4,5]$.

El río Manzanares, ubicado en el departamento del Magdalena, es una de las fuentes de agua dulce más importantes de la ciudad de Santa Marta [6-10]. Entendiendo la necesidad latente de contar con un SAT, la Universidad Cooperativa ha desarrollado un proyecto que busca, a través de una red, sensores ubicados en el río y la implementación de tecnología de vanguardia. De manera que, al conocer el estado del río en todo momento, se logre alertar de forma oportuna a los usuarios vulnerables, además de activar los protocolos de emergencia definidos por las entidades encargadas del riesgo en la ciudad y, así, mitigar cualquier daño que pueda ocasionarse por este.

\section{SISTEMAS DE INFORMACIÓN}

$\square$ i observamos la definición formal dada por Andreu, Ricarte y Valor [11], un sistema de información es un: "conjunto formal de procesos que, operando sobre una colección de datos estructurada de acuerdo a las necesidades de la empresa, recopila, elabora y distribu- 
yen selectivamente la información necesaria para la operación de dicha empresa y para las actividades de dirección y control correspondientes, apoyando, al menos en parte, los procesos de toma de decisiones necesarios para desempeñar funciones de negocio de la empresa de acuerdo con su estrategia".

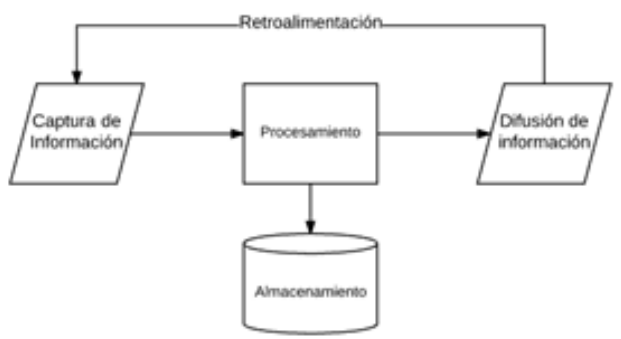

Figura 1. Sistema de información

Debemos agregar al respecto que los sistemas de información han sido la herramienta clave para afrontar los retos de la nueva era en las empresas, contribuyendo a la expansión y llevando un control preciso de procesos y de información, Sin importar las barreras geográficas o de idioma, los sistemas de información pueden ser usados para optimizar cualquier proceso, dado que todo proceso conlleva al manejó de información, y dichos sistemas buscan el manejo eficaz de esta, logrando así mejores resultados en todas las áreas implementadas, como por ejemplo, servicio al cliente, manejo de movimientos financieros, manejo de inventarios, entre muchos más.

Los sistemas de información deben ser conformados por los siguientes elementos macros: Entrada de Información, Almacenamiento de información, el Procesamiento de la información, Salida de Información; dichos elementos conforman el esquema básico de un sistema de información, donde cada elemento cumple una función primordial (Figura 1).

\section{SISTEMA DE ALERTA TEMPRANA}

Oegún las Naciones Unidas [12], el SAT es el conjunto de herramientas $\checkmark y$ acciones necesarias para difundir en forma eficaz información de alerta que logre la mitigación total o parcial de los daños. Dada esta definición, nuestro proyecto es enteramente un sistema de alerta temprana aplicado a la ciudad de Santa Marta.

\section{A. Niveles de alertas en Colombia}

En Colombia, los niveles de alerta son definidos por el IDEAM [13], pero cada municipio puede emitir sus propios niveles de riesgo si se encuentran frente a una emergencia fluvial. En general, se definen tres niveles de alerta: la alerta de nivel amarilla, que se declara cuando la intensidad de lluvias pueda causar inundaciones en los siguientes días; la alerta naranja, cuando se denota que la tendencia del nivel del es al alza y se pueden presentar inundaciones en horas próximas; por último, la alerta roja, cuando el nivel del río alcanza niveles críticos y se hace inminente una inundación o ya se ha iniciado una inundación.

\section{B. Mitigación}

Este concepto es definido por la Naciones Unidas [12] como la disminución de daños causados por un desastre o por una amenaza sobre una comunidad vulnerable. Esto se logra a través de un plan y la implementación de herramientas o planes de evacuación realizados para poblaciones ubicadas en el área de afectación de dicha amenaza. 
PORTAL WEB Y APP ANDROID

$\mathrm{E}$ n el caso del SAT río Manzanares, se han desarrollado las primeras versiones de un portal Web y una aplicación Android, los cuales apoyan el proceso de recolección de información de la red de sensores, el análisis de los datos y la emisión de alertas para la población afectada y entes de control relacionados. La aplicación web fue creada a partir de la tecnología framework JSF, aplicando el API EJB que utiliza el código java para su programación, además de Hibérnate, como librería de persistencia de datos. Glassfish fue el servidor seleccionado para la administración de las conexiones de los web Services que trasmiten información desde los sensores físicos. Por otro lado, se cuenta con una aplicación móvil, desarrollada bajo la herramienta Android Studio y la estructura de desarrollo Android que implementan java y xml. Además, se realizó la implementación del servicio de GCM (Google Cloud Messaging), el cual permite enviar alertas push a cualquier dispositivo Android.

Para ingresar a las opciones del sistema, se debe contar con un usuario registrado, dicho nombre es único en el sistema y no lleva espacio, el resto de campos son igualmente obligatorios en el sistema.

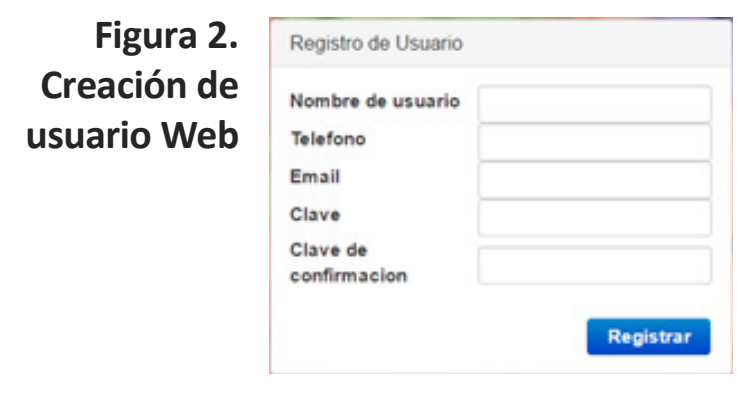

En la autenticación, para ingresar al sistema, los usuarios deben digitar su nombre de usuario y su contraseña, luego presionar el botón "Entrar" para realizar el login. Si los datos son correctos, seguirá a la vista de inicio.

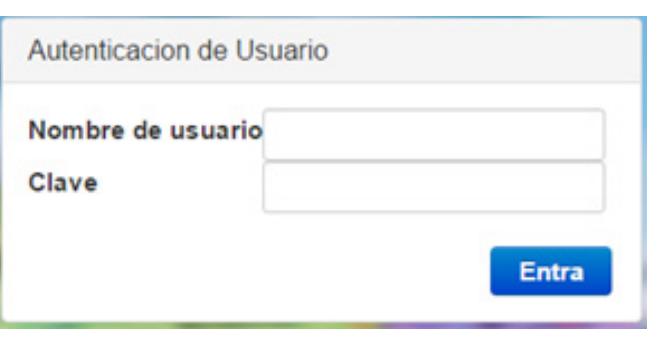

Figura 3. Loging Web

- $\quad$ Edición de perfil de usuario Luego de autenticarse, el sistema redirige al usuario a la página de inicio con el menú de las opciones. En el lado derecho superior de este, se hallan las opciones de edición de perfil de usuario.

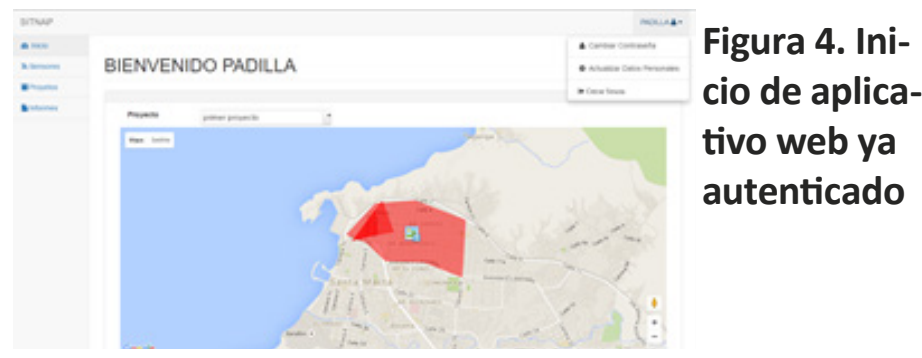

En este módulo podemos editar nuestros datos personales, entendiendo que el nombre de usuario no se va cambiar ya que es único en el sistema.

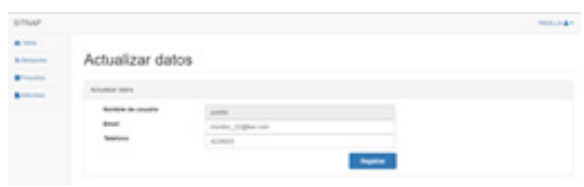

Figura 5. Edición de perfil de usuario

\section{- $\quad$ Cambio de contraseña}

Los usuarios pueden cambiar de contraseña las veces que deseen, ya que con esto pueden tener una cuenta segura, como se indica en la imagen pertinente. 


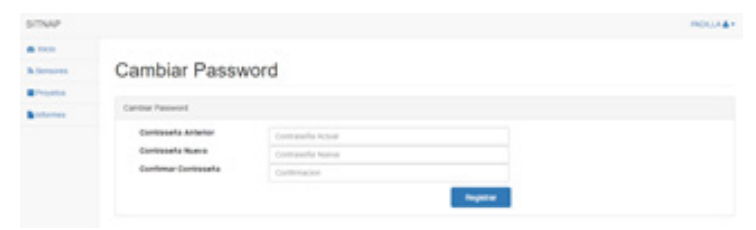

- Gestión de proyecto, Registro de tipo de sensor

Para crear un proyecto, hay que crear primero los tipos de sensores, el cual lleva un icono de 32x32 pixeles. Además de definir una unidad de medida y un nombre.
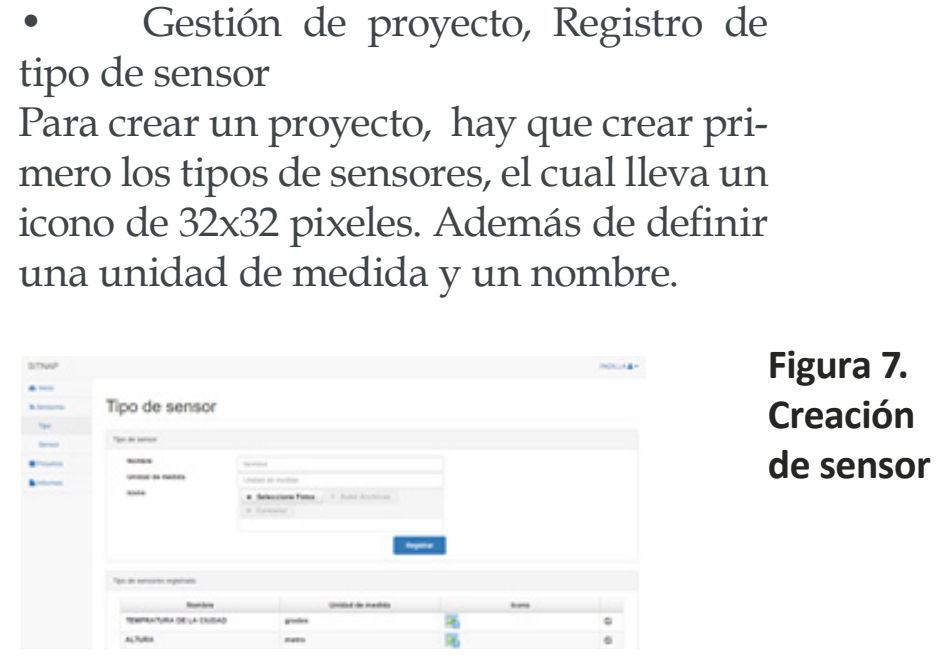

- $\quad$ Registro de proyecto

Ya creado los tipos de sensores se procede a crear proyectos en forma propiamente dicha. Para esto se selecciona un tipo de sensor y se van adicionando dispositivos según la naturaleza de los sensores que se vayan a usar. Se pueden crear sensores nuevos o sensores ya creados de otros proyectos, pero tal sensor solo existe cuando el proyecto donde inicialmente se va a usar, es creado.

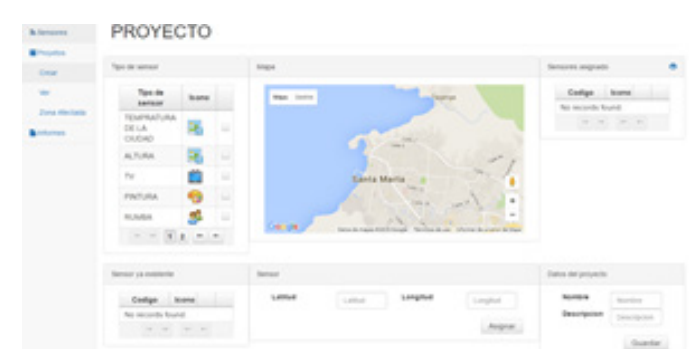

Los sensores son las entidades principales con las que se crean los proyectos y siguen dos procesos automatizados: el dado de alta y el envío de información. A continuación, se muestran dos flujogramas que explican los procesos anteriormente mencionados:

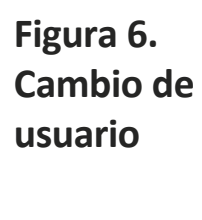

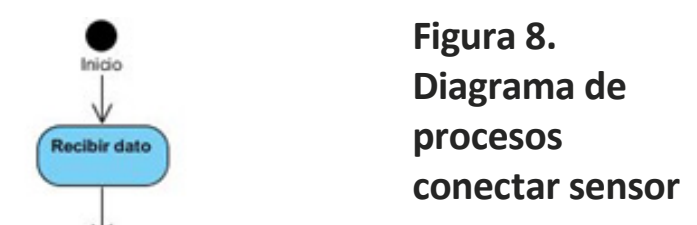

133

Figura 9.

Diagrama de procesos enviar dato sensor
- Dibujar zona afectada

Esta funcionalidad busca sombrear la zona de afectación de inundación o de cualquier desastre que se esté midiendo. Para esto se agregan puntos para dibujar polígonos del proyecto en cuestión, pudiéndose agregar todos los marcadores que se desee, pero para dibujar la forma en el mapa, estos marcadores deben ser más de dos.

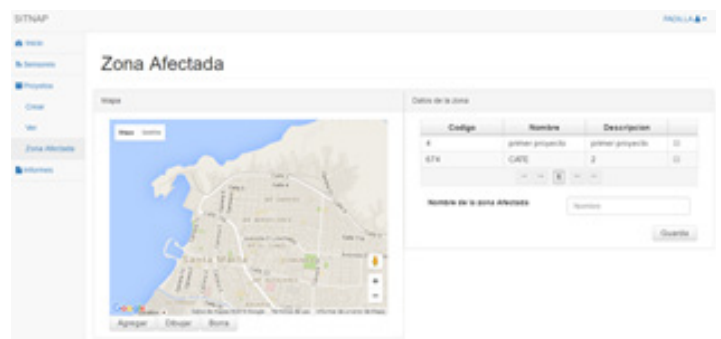

Figura 10. Zona afectada 
La aplicación Android cuenta con un listado general de proyectos, de donde el usuario ha de seleccionar los de su preferencia y adicionarlos a una lista personal.

\section{Figura 11. Selección de proyecto}

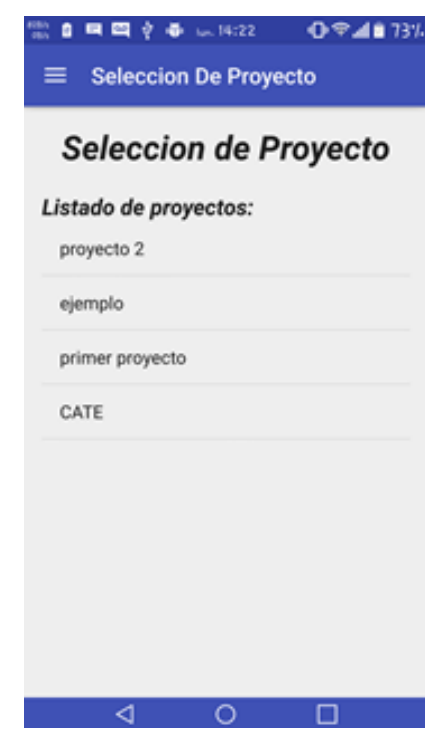

134 Cada proyecto debe contar con una vista, donde se mostrarán las zonas de afectación al igual que la posición de los sensores, a fin de activar o desactivar las alertas asociadas al proyecto.

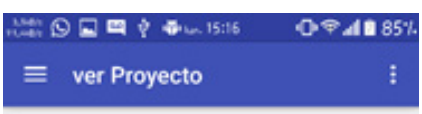

Figura 12.

$$
\text { proyecto } 2
$$

Zona de localización

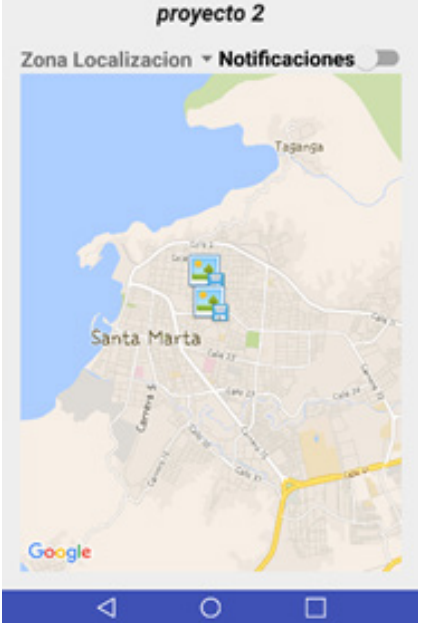

Una de las funcionalidades más destacadas con las que cuenta el aplicativo es la recepción de las alertas. Esta funcionalidad se implementa con la ayuda del ser- vicio de Google Cloud Messaging y permite enviar una notificación a la pantalla del dispositivo en cualquier momento, sin importar si la aplicación está siendo ejecutada. Se efectúa cuando en un proyecto específico el usuario activa el switch de notificación:

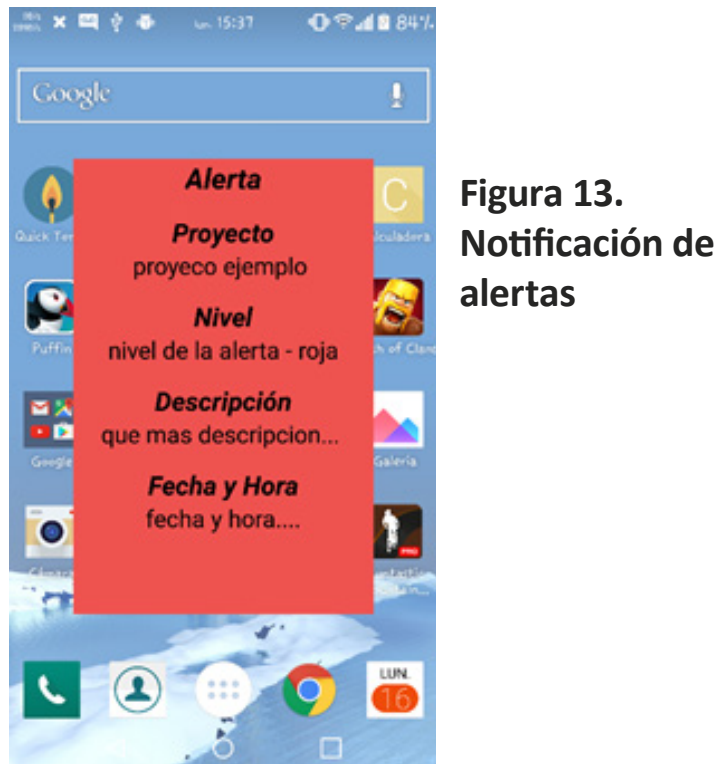

\section{CONCLUSIONES}

T

os sistemas de alerta temprana juegan un papel importante en la gestión del riesgo de los centros de operaciones de emergencia. En la ciudad de Santa Marta no se ha implementado un sistema que permita interpretar las variables humedad, nivel de agua y precipitaciones, y con ellas establecer una alerta temprana de inundación del Río Manzanares. Por esta razón, el grupo de investigación ISI de la Universidad Cooperativa de Colombia, sede Santa Marta, elaboró un sistema de alerta que hemos presentado en este trabajo y que a la fecha integra las siguientes herramientas y dispositivos tecnológicos:

- $\quad$ Plataforma web de manejo de proyectos de censado, análisis de información y divulgación de la alerta. 
- $\quad$ Aplicativo Android de divulgación general de la alerta y consulta de proyectos de medición.

\section{Referencias Bibliográficas}

[1] OEA DDS, Manual para el diseño, instalación, operación y mantenimiento de sistemas comunitarios de alerta temprana, 2010. [En línea]. Disponible en: http://www.rimd.org/advf/documentos/4ce2ae1f4aa62.pdf. [Último acceso: 15 Agosto 2013].

[2] J. Pollner, J. Kryspin-Watson y S. Nieuwejaar. Disaster Risk Management and Climate Change Adaptation in Europe and Central Asia. Washington, D. C: World bank Group, 2008.

[3] V. V. Krzhizhanovskaya, N. B. Melnikova, A. M. Chirkin, S. V. Ivanov, A. V. Boukhanovsky y P. M. A. Sloot. “Distributed simulation of city inundation by coupled surface and subsurface porous flow for urban flood decision support system", Procedia Computer Science, pp. 1046-1056, 2013.

[4] B. Balis, M. Kasztelnik, M. Bubak, T. Bartynsk, T. Gubała, P. Nowakowski y J. Broekhuijsen. "The UrbanFlood Common Information Space for Early Warning Systems,» Procedia Computer", Science, vol. 4, p. 96-105, 2011.

[5] URBANFLOOD CONSORTIUM 2015, Urbanflood, 2012. [En línea]. Disponible: http://www.urbanflood.eu/Pages/ default.aspx. [Último acceso: 2 Junio 2014]. [6] EL Tiempo, eltiempo.com, 2010. [En línea]. Disponible: http://www.eltiempo. com/archivo/documento/CMS-7876699.

[7] El Tiempo, eltiempo, 2004. [En línea]. Disponible: http://www.eltiempo. com/archivo/documento/MAM-1544397.

[8] D. Piraquive, danielpiraquive. blogspot.com, 2011. [En línea]. Disponible: http://danielpiraquive.blogspot.com. co/2011/11/las-malvinas-se-preparan-para-los.html.

[9] reliefweb, 2010. [En línea]. Disponible: http://reliefweb.int/report/colombia/ colombia-70-muertos-y-661-mil-afectados-deja-primera-temporada-invernal-del-a\%C3\%B1o-en.

[10] Diario Norte, 2011. [En línea]. Disponible en: http://www.diarionorte.com/ article/70153/ocho-muertos-y-siete-desaparecidos-por-lluvias-en-colombia-.

[11] A. H. Trasobares, Los sistemas de información: evolución y desarrollo, [En línea]. Disponible en: http://dialnet.unirioja.es/descarga/articulo/793097.pdf.

[12] Naciones Unidas, Terminología sobre Reduccion del riesgo de desastres, Ginebra, 2009.

[13] IDEAM - Instituto de Hidrología,

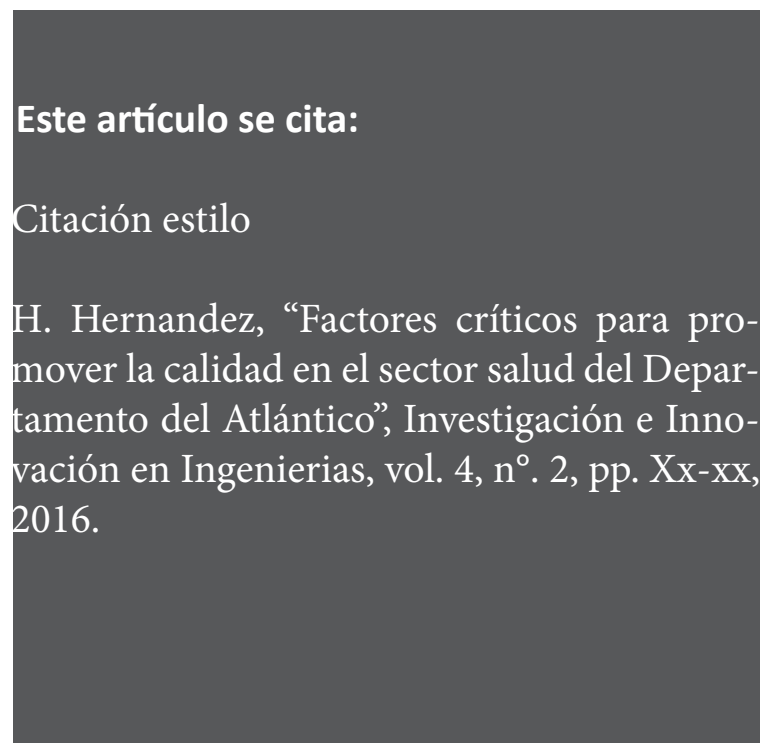

